\title{
Le Congrès des américanistes de Nancy en 1875 : entre succès et désillusions
}

\section{Étienne Logie et Pascal Riviale}

\section{(2) OpenEdition}

\section{Journals}

Édition électronique

URL : https://journals.openedition.org/jsa/11036

DOI : 10.4000/jsa. 11036

ISSN : 1957-7842

\section{Éditeur}

Société des américanistes

\section{Édition imprimée}

Date de publication : 5 décembre 2009

Pagination : 151-171

ISSN : 0037-9174

\section{Référence électronique}

Étienne Logie et Pascal Riviale, «Le Congrès des américanistes de Nancy en 1875 : entre succès et désillusions », Journal de la Société des américanistes [En ligne], 95-2 | 2009, mis en ligne le 10 décembre 2014, consulté le 03 septembre 2022. URL : http://journals.openedition.org/jsa/11036 ; DOI : https://doi.org/10.4000/jsa. 11036 


\title{
LE CONGRÈS DES AMÉRICANISTES DE NANCY EN 1875 : ENTRE SUCCÈS ET DÉSILLUSIONS
}

\author{
Étienne LOGIE et Pascal RIVIALE *
}

Le 19 juillet 1875 s'ouvre à Nancy le premier congrès international des américanistes, dans le prestigieux palais ducal. Ses initiateurs, la Société d'Ethnographie et la Société Américaine de France, ont su travailler de concert avec un comité local d'organisation, remarquablement dirigé par deux personnalités nancéiennes : le baron Guerrier de Dumast et Lucien Adam. Le congrès connaît un vif succès qui en appellera d'autres. Mais des ambitieux projets américanistes conçus pour la cité ducale, il ne reste plus que quelques pièces de collections éparpillées, pour lesquelles bien des questions restent encore sans réponse, ainsi que quelques lointains souvenirs dus à la commémoration à Nancy du centenaire de ce premier congrès. [Mots-clés : congrès, Nancy, Guerrier de Dumast, Lucien Adam, Musée américain, Société d'Ethnographie, Société Américaine de France, américanistes.]

The Nancy congress of Americanists (1875) : successes and disappointments. The first international congress of Americanists opened on July 19th 1875 and was held in the prestigious palace of the Dukes of Lorraine, Nancy. Its instigators, the Société d'Ethnographie and the Société Américaine de France worked in close collaboration with a local board of organization, remarkably led by two prominent notables of Nancy : the Baron Guerrier de Dumast and Lucien Adam. The congress was a tremendous success and opened up a great and vivid tradition. However, little remains of the ambitious projects concerning American studies which were planned for Nancy : a few scattered museum pieces, asking many unanswered questions, and some distant memories recovered during the celebration of centenary of the first Nancy congress. [Key words : congress, Nancy, Guerrier de Dumast, Lucien Adam, Musée américain, Société d'Ethnographie, Société Américaine de France, americanists.]

El congreso de Nancy (1875) : entre éxito y desilusiones. El 19 de julio de 1875 se abre el primer Congreso international de americanistas en el prestigioso Palacio ducal. Sus iniciadores, la Société d'Ethnographie y la Société Américaine de France, supieron trabajar de común acuerdo con un comité local organizador, muy bien dirigido por dos personalidades de Nancy : el barón Guerrier de Dumast y Lucien Adam. El Congreso conoce un gran éxito llamado a desembocar en otros. Sin embargo, de los ambiciosos

* CRULH-EA 3945, Université Nancy 2, 3 place Godefroy de Bouillon 54000 Nancy [hglogie@yahoo.fr] ; Archives nationales, 60 rue des Francs-Bourgeois 75003 Paris et Centre EREA (UMR 7186, CNRS, Villejuif) [pascal.riviale@culture.gouv.fr].

Journal de la Société des Américanistes, 2009,95-2, pp. 151-171. (C) Société des Américanistes. 
proyectos americanistas concebidos para la ciudad ducal, subsisten sólo unas pocas piezas de colección diseminadas, sobre las cuales muchas preguntas quedan sin respuestas, y algunos recuerdos reactivados por la conmemoración en Nancy del centenario de este primer congreso. [Palabras claves: congreso, Nancy, Guerrier de Dumast, Lucien Adam, Musée américain, Société d'Ethnographie, Société Américaine de France, americanistas.]

Le 19 juillet 1875 s'ouvre à Nancy le premier congrès international des américanistes. Cette réunion scientifique se place sous le patronage de la Société d'Ethnographie. Pour la première fois, des « savants des deux mondes » viennent débattre des questions de fond du champ d'étude américaniste. Si cette manifestation est de nos jours bien enracinée dans le paysage scientifique, on peut s'interroger sur les facteurs ayant rendu possible cette grande première. En ce dernier quart du XIX ${ }^{\mathrm{e}}$ siècle, dans un contexte de grande dispersion de la recherche américaniste, la tenue d'une telle réunion paraissait une gageure. Pourtant, il a non seulement été possible de l'organiser, mais les résultats ont été jugés suffisamment probants par ses participants pour que l'on souhaite renouveler l'expérience, puis l'instituer en une manifestation régulière. Il convient donc de s'interroger sur l'impact de cette première session, tant sur la sociabilité américaniste à l'échelle internationale que sur les activités nancéiennes dans ce champ d'études spécifique.

\section{L'ORGANISATION DU CONGRÈS}

\section{Pourquoi un tel congrès?}

Les origines du congrès international des américanistes sont à replacer dans un contexte alors nouveau dans l'histoire des sciences. À l'instar des disciplines scientifiques déjà bien établies, on observe dans la seconde moitié du XIX ${ }^{\mathrm{e}}$ siècle une volonté de rendre des domaines de recherches plus visibles, non seulement auprès des spécialistes, mais aussi d'un plus large public ; de même, les échanges internationaux entre confrères tendent à se formaliser. Pour les champs d'études encore jeunes - en l'occurrence tous ceux relevant des sciences de l'homme et de la société (l'archéologie préhistorique, l'anthropologie, l'ethnographie...) -, il fallait gagner une crédibilité et obtenir une reconnaissance oflicielle et publique en tant que sciences véritables, au même titre que la botanique ou la chimie, par exemple. Dans cette perspective, les rencontres internationales offraient la possibilité de développer une réflexion commune entre spécialistes sur ce que devait être le champ d'action d'une discipline donnée, s'accorder sur un certain nombre de références et d'objectifs, voire sur les limites du domaine, d'échanger points de vues et informations sur l'état de la recherche ou sur des questions particulières, et de développer des réseaux de contacts. Ces rencontres étaient aussi un moyen de 
gagner en visibilité auprès du public ${ }^{1}$. On observe la tenue d'un nombre croissant de congrès internationaux à partir des années 1860 . Après une première rencontre à La Spezia (Italie) en 1865, puis à Neuchâtel en 1866, les spécialistes de l'archéologie et de l'anthropologie préhistorique inaugurent à Paris en 1867 la tenue régulière de leur congrès international. C'est en 1873 que Léon de Rosny et quelques autres membres de la Société d'Ethnographie lancent l'idée d'une rencontre des spécialistes de l'orientalisme au niveau international. Le succès de cette réunion aboutit à l'instauration d'un cycle régulier de rencontres tant internationales que nationales. Le modèle de ce type d'événement ne pouvait qu'être séduisant pour tous ceux qui rêvaient de voir un jour l'américanisme acquérir une reconnaissance oflicielle autorisant son institutionnalisation. Les multiples initiatives associatives de la Société d'Ethnographie illustrent ce profond souci de sociabilité et de fraternité internationale, volontiers exprimé dans les discours. Fondée en 1859 par Léon de Rosny, Brasseur de Bourbourg, Charles de Labarthe et Edme Jomard, la Société d'Ethnographie Orientale et Américaine ${ }^{2}$ s'investit d'une triple mission : définir les paradigmes de l'ethnographie ; centraliser et promouvoir les travaux - notamment d'archéologie - des " rares savants adonnés à l'étude de l'Amérique antécolombienne ${ }^{3}$ »; assurer la promotion et la vulgarisation des études orientales. Après une première tentative de séparation en 1863, le Comité d'Archéologie Américaine avait réintégré la Société d'Ethnographie; en 1873, il gagna finalement son émancipation sous le nom de Société Américaine de France.

En fait, autant l'orientalisme paraît avoir trouvé assez tôt dans le $\mathrm{XIX}^{\mathrm{e}}$ siècle son ancrage dans le paysage scientifique français, autant on peut constater dans le même temps les grandes difficultés rencontrées pour faire admettre le Nouveau Monde comme un objet d'étude à part entière. Pourtant, la multiplication des découvertes de vestiges architecturaux et d'artefacts parfois spectaculaires, le nombre croissant des explorations, l'enrichissement incessant des collections archéologiques et ethnographiques américanistes dans les musées européens, les recherches entreprises pour décrypter les sources vernaculaires écrites et orales, contribuèrent à constituer un savoir sur l'Amérique indigène qui ne demandait qu'à se développer et à être approfondi, pour peu que des spécialistes s'y consacrassent. Désormais, ce n'est plus seulement en Europe, mais également en Amérique du Nord et du Sud que se manifeste une volonté grandissante de mener des recherches sur les populations autochtones - présentes ou disparues -, reposant sur des critères scientifiques. Dans le dernier quart du XIX ${ }^{\mathrm{e}}$ siècle, les conditions paraissent donc nettement plus favorables à l'émergence d'un champ d'étude propre aux Amériques. Avec la (re)création de la Société Américaine de France en 1873 à Paris, les américanistes issus de la Société d'Ethnographie pensent que leur heure est venue et espèrent pouvoir mobiliser un mouvement international en ce sens. Un projet précurseur aurait été envisagé dès 1867 par quelques membres de la première société américaniste - le Comité d'Archéologie 
américaine, déjà mentionné -, autour de Martin de Moussy et de William Bollaert, lors d'un voyage à Londres (Riviale 1996). Toutefois le projet ne devait pas voir le jour, et c'est certainement dans la dynamique de la réussite du premier congrès international des orientalistes, tenu à Paris en 1873, qu'est remise à l'ordre du jour l'idée d'une rencontre du même type pour les américanistes. Le congrès des orientalistes avait été organisé sous l'égide de la Société d'Ethnographie (et présidé par Léon de Rosny). Celui des américanistes le sera sous le double patronage de la Société Américaine de France et d'un comité organisateur à Nancy (présidé par le Nancéien Guerrier de Dumast), sur le modèle du congrès des orientalistes ${ }^{4}$. Du fait de la proximité entre institutions, tous les membres de la Société d'Ethnographie (d'où sont issus les regroupements orientaliste et américaniste) sont sollicités pour s'inscrire au congrès des américanistes à venir et bénéficient naturellement d'un tarif préférentiel.

\section{Un congrès en province}

La question scientifique ne saurait être suffisante pour justifier un exercice aussi ambitieux. Depuis de nombreuses années, les américanistes s'efforçaient, en vain, de développer à Paris un centre spécialisé dans leurs études, mais certains d'entre eux, tels que Rosny ou d'André de Clavery, avaient apparemment le sentiment d'être incompris, voire dédaignés, par les milieux académiques offìiciels. Dès lors, la poursuite de leur œuvre en province apparut comme une solution. À cela s'ajoute une dimension politique importante. Les membres des grandes sociétés savantes sont, pour certains d'entre eux, en étroite relation avec le monde politique. Ils sont sensibles aux grandes questions de fond présentes dans tous les courants politiques, ainsi qu'à celles qui les transcendent. La question de la décentralisation alimente nombre de débats dans les cercles de l'élite sociale française. La Société d'Ethnographie et son entourage vont enfourcher ce cheval de bataille. Peut-être dès la fin du premier congrès des orientalistes (Paris, 1873), il est envisagé de doubler les réunions internationales de sessions provinciales. La première allait avoir lieu à Saint-Étienne en septembre 1875. Voici quel était l'argumentaire accompagnant l'invitation à cette session :

En France le public s'intéresse peu, trop peu même aux études orientales [...]. Il faut rechercher particulièrement la cause dans la trop grande centralisation de l'orientalisme et dans l'insuffisance de publicité [...]. L'Athénée [oriental, section de la Société d'Ethnographie créée en 1864, puis recréée en 1874] ne fait donc pas seulement appel à Paris pour sa session de Saint-Étienne, c'est-à-dire aux illustrations, aux maîtres de la science, aux professeurs de nos grandes institutions nationales (les gloires de l'orientalisme français !), mais aussi à la Province, c'est-à-dire [...] aux savants et aux amateurs encore inconnus du public, qui prendraient des places distinguées dans le domaine de l'érudition, s'ils voulaient faire connaître leurs travaux inédits ou en préparation. (Textor de Ravisi 1875 , pp. 8-11) ${ }^{5}$ 
Le rapport du comité de direction de la session inaugurale du congrès des orientalistes, rédigé par Victor Dumas, alors secrétaire adjoint de la Société d'Ethnographie, va dans le même sens pour justifier la tenue du congrès des américanistes en province : « la Société d'Ethnographie, poursuivant un système d'idées décentralisatrices, a voulu prouver que la province française pouvait faire fructifier une pensée tout aussi bien que Paris, et elle a confié à la généreuse Lorraine le soin de lui donner raison ${ }^{6}{ }^{6}$. Pour beaucoup d'entre eux, les événements culturels et scientifiques doivent servir de vitrine à leurs thèses politiques.

\section{Le choix de Nancy}

Le choix de Nancy semble avoir été fait par le bureau de la Société Américaine de France (ci-dessous désigné sous l'acronyme de SAF). Il repose surtout sur quatre éléments. D'abord, Nancy est l'un des grands centres des études orientalistes en France. D'éminents orientalistes sont nancéiens à l'image de Burnouf et de Leupol ${ }^{7}$, ou encore de Lucien Adam. Avec Guerrier de Dumast, l'orientalisme connaît à Nancy un dynamisme puissant qui œuvre pour l'ouverture d'une chaire de langues orientales à la faculté de lettres au cours des années 1850 à 1870 . Cette position particulière est soutenue par des personnalités telles que Foucaux, Eichoff, Léon de Rosny, Chavée et Oppert. Tous ces membres de la Société d'Ethnographie deviennent membres correspondants nationaux de l'Académie de Stanislas au cours de cette période. Un autre objectif, découvert récemment, de ce groupe provincial d'orientalistes résonne avec les vœux de la Société d'Ethnographie : vulgariser l'étude des langues orientales, en particulier le sanscrit et l'arabe. Bien que mise en concurrence avec d'autres villes comme Nantes ou Bordeaux (Logie 2008, p. 35), Nancy apparaît donc aux initiateurs du congrès comme un choix qui s'impose. Ensuite, la question de la décentralisation a connu un puissant écho à Nancy où le fameux « Programme de Nancy » avait été rédigé en 1865. Celui-ci suscite un large débat dans les sphères politiques nationales et lorraines. Il reçoit un appui enthousiaste d'hommes politiques tels que Jules Ferry, Sadi Carnot, Casimir Périer, Guizot ou Falloux. Ce mouvement est entendu par Napoléon III qui crée, en janvier 1870, une commission sur la décentralisation, composée notamment de Lorrains ${ }^{8}$. Nancy se trouve donc au cœur de débats politiques importants dans les années 1860-1870. Les intellectuels nancéiens ont démontré leur capacité d'action et se font fort des soutiens reçus à cette occasion. Puis, Nancy symbolise la France meurtrie par l'humiliation de la guerre de 1870 contre la Prusse qui eut pour conséquence la perte de l'AlsaceMoselle. Les troupes allemandes occupent la cité lorraine jusqu'en 1873, renforçant le sentiment de méfiance, voire de revanche armée, des Français à l'endroit du Reich allemand. D'un autre côté, l'organisation d'une réunion scientifique rassemblant des savants issus des deux mondes peut illustrer une forme d'idéal pacifiste et de réconciliation. Dans l'esprit de ses organisateurs, le poids de 
l'histoire doit marquer ce premier congrès: une ville forte d'un riche passé s'impose ainsi logiquement. Nancy offre, par ailleurs, un sérieux indiscutable. Celui-ci est renforcé par la prise de position de l'Allemagne, grande nation scientifique à cette époque, qui demande la tenue de cette manifestation dans l'une de ses villes (Logie 2008, p. 35). Dans le contexte, la demande allemande était difficile à accepter du côté français. En revanche, Nancy, ville frontalière juste libérée des troupes d'occupation allemande, présente un profil séduisant et a l'avantage de ne pas froisser l'Allemagne, puissance européenne de premier ordre. Nancy devient alors un double symbole.

Enfin, la présence de la prestigieuse Académie de Stanislas offre aux initiateurs du congrès une caution scientifique et la possibilité d'exploiter un important réseau de relations. En effet, celle-ci est en contact avec quelques grandes institutions et revues scientifiques, à l'instar de l'Académie française, l'Association scientifique de France, la Smithsonian Institution. Parmi les membres de l'Académie, deux personnalités se détachent: le baron Guerrier de Dumast et le magistrat Lucien Adam. Le premier est correspondant de l'Institut et ami du président de la République. C'est aussi un membre très actif de la loge maçonnique des artistes du Grand Orient à Paris, puis à Nancy. Le second a aussi tissé un important réseau d'amitiés tant en France qu'en Amérique au cours de ses pérégrinations. Adam (1874) a participé au premier congrès international des orientalistes de 1873, dont il dresse un résumé lors de son discours de réception à l'Académie de Stanislas le 15 janvier 1874. Nous disposons de peu d'informations sur la période qui précède l'annonce de la tenue du congrès à Nancy en octobre 1874. Toutefois, le discours d'Adam laisse penser que le choix de Nancy a pu être envisagé assez tôt. Adam a peut-être entendu parler d'un projet de réunion américaniste en province. Compte tenu de son caractère d'homme engagé, il se serait alors employé pour promouvoir Nancy auprès des instances parisiennes et del'Académie de Stanislas.

\section{LA TENUE DU CONGRÈS DE NANCY}

\section{Le Musée lorrain accueille des congressistes venus du monde entier}

Le comité de la SAF rédigea le 25 août 1874 les statuts du congrès de Nancy, calqués sur ceux du congrès des orientalistes. Le 22 juillet 1875 , sur la proposition de Léon de Rosny et/ou de Madier de Montjau, le bureau du congrès en avait décidé ainsi : lesdits statuts avaient fait la preuve de leur efficacité. À l'instar du congrès des orientalistes, celui des américanistes est financé grâce à des fonds propres uniquement. Seules les cotisations et quelques dons (comme ceux du président Mac-Mahon, du ministère de l'Instruction publique ${ }^{9}$ et de la chambre du commerce et de l'industrie de Nancy) permettent au congrès de fonctionner. À l'ouverture du congrès, 1486 souscripteurs français, étrangers et lorrains sont 
enregistrés. Ces derniers comptent 724 personnes soit 48,7\%. On dénombre 440 autres souscripteurs pour le reste de la France et 322 pour l'étranger. Le succès financier du congrès repose donc essentiellement sur la remarquable participation des souscripteurs lorrains. Les membres du comité de Nancy sont pour la plupart des magistrats, des membres de l'enseignement supérieur ou des ecclésiastiques. Les magistrats représentent près de $20 \%$ des souscripteurs, les enseignants en Faculté environ $10 \%$ et les membres de l'Église $6 \%$. Il faut y ajouter les militaires à hauteur de $5 \%$. Il s'agit ici des souscriptions reçues jusqu'au matin du 19 juillet 1875 (date de l'ouverture du congrès). La liste des souscriptions demeure ouverte encore quelques temps, au moins jusqu'à la mi-août 1875. De ce fait, ce sont 1795 cotisations qui arrivent aux comités d'organisation au cours de la période globale de souscription. La réussite de la réunion entraîne probablement un regain d'intérêt, justifiant ainsi le nombre conséquent de cotisations tardives, y compris en Lorraine.

On observe que le nombre global s'accroît, avec des différences selon les catégories. La répartition entre Français et étrangers s'équilibre au cours de la période indiquée (Figure 1). Le groupe des Français est celui qui gagne le moins de nouveaux adhérents avec seulement 48 nouvelles inscriptions entre juillet et août. Les cotisations étrangères augmentent fortement avec 145 nouveaux inscrits. Si la plupart des délégations étrangères proposent des groupes de 4 à 8 cotisants (à l'image du Brésil : 5, de l'Espagne : 4 ou du Salvador : 4), d'autres délégations sont plus nombreuses ${ }^{10}$. Les plus importantes sont le Canada (33), les États-Unis (32), le Pérou (26), le Mexique (21), le Japon (19). On remarque ici la forte représentation de l'Amérique du Nord. Le cas du Canada est révélateur de la composition générale des délégations. On y retrouve huit membres de l'Église, six membres de l'éducation, six avocats. Les autres membres sont des hommes politiques, des hommes de lettres et des militaires. La présence de

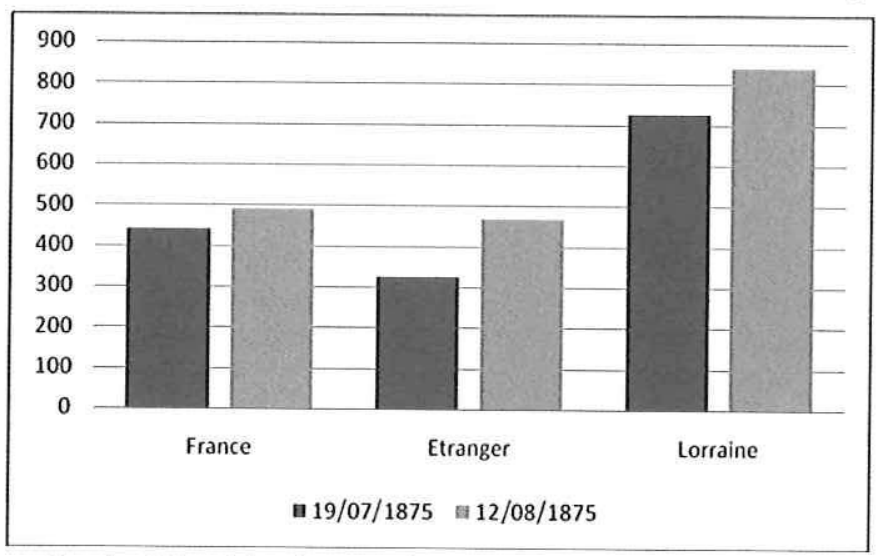

FIG. 1 - Répartition des souscripteurs au congrès de Nancy (1875). 
certains pays d'Asie (comme le Japon) rappelle l'influence des milieux orientalistes dans l'organisation du congrès. Les Lorrains sont, quant à eux, 116 de plus. Si la Lorraine conserve une place prépondérante, l'augmentation de la part des étrangers est, elle, essentielle pour expliquer l'impact que l'événement a eu à l'extérieur de la France. De fait, les représentants des délégations étrangères présents à Nancy ont dû faire part du succès du congrès de sorte qu'il devint fondateur, en étant reconduit à Luxembourg en 1877. C'est là un phénomène capital dans le processus de pérennisation des grandes réunions. La répartition des catégories socioprofessionnelles demeure dans les mêmes proportions. Le congrès de Nancy concerne surtout les milieux aisés et intellectuels. Il ne s'agit, en aucun cas, d'une manifestation populaire. Néanmoins, tous les souscripteurs ne participèrent pas physiquement aux activités de ce rassemblement. Le nombre de congressistes se situe entre 1040 et 1120 personnes (soit environ $60 \%$ de l'ensemble des personnes ayant versé une souscription). Il convient de rappeler que ces premières réunions scientifiques sont encore avant tout des rendez-vous de sociabilité d'un nouveau genre: près de la moitié des personnes se sont contentées d'exprimer leur soutien à l'initiative en ne faisant que payer une souscription, et un grand nombre d'autres viendra assister au congrès sans jamais prendre la parole. Les véritables acteurs de cette réunion internationale ne représentent encore qu'une petite minorité de l'ensemble des inscrits.

\section{La place des sociétés savantes nationales initiatrices}

Regardons à présent de plus près le rôle de la Société d'Ethnographie et de la SAF. Aucun souscripteur lorrain, à l'exception de quelques membres du comité d'organisation, n'est membre de l'une ou de l'autre de ces sociétés au moment de la publication des actes du congrès. La Société d'Ethnographie apporte 213 souscripteurs, équitablement répartis entre la France et l'étranger (Figure 2). Au total,

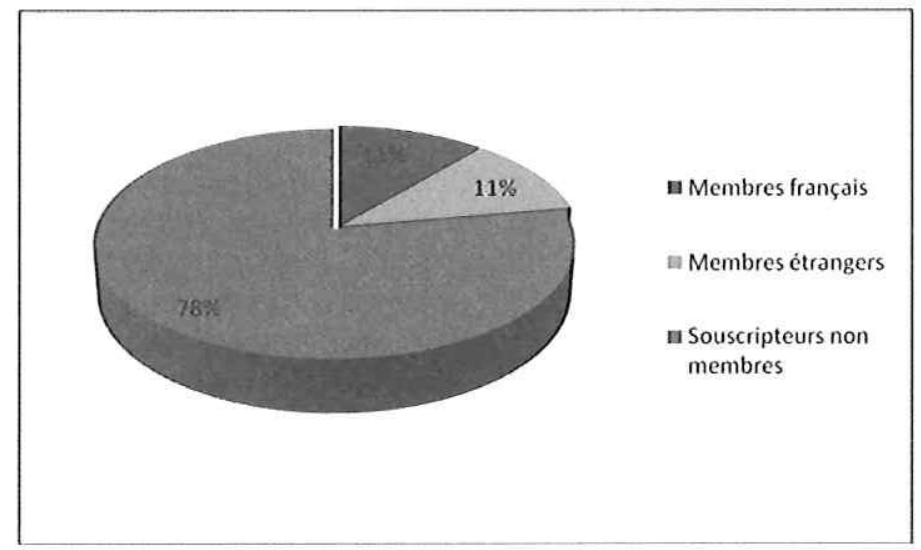

FIG. 2 - Part des membres de la Société d'Ethnographie dans les souscriptions - hors Lorraine. 
ceux-ci fournissent $22 \%$ des souscriptions. Cela en fait un groupe non négligeable, preuve de l'implication de l'association dans cet événement.

Cinquante trois sociétaires de la SAF souscrivent au congrès, montrant sa relative modeste influence en ce domaine (Figure 3). Il ne faut pas oublier par ailleurs qu'il existe une grande porosité entre la SAF et la Société d'Ethnographie. Des américanistes s'inscrivent de fait en tant que membres de la Société d'Ethnographie (qui bénéficie d'un tarif préférentiel de six francs). Encore soumise à son aînée, la SAF ne dispose pas en réalité, à ce moment, de moyens suffisants pour peser davantage sur le congrès, à la différence de ce qui se posera ensuite. Au final, les membres de l'une ou l'autre de ces sociétés constituent une part notable des souscripteurs étrangers et nationaux ( $28 \%$ du total). Mais ces sociétés apportent aussi leur soutien par leur renom et par l'engagement de personnalités comme Boban, Bollaert, Cernuschi, Darwin, Méhédin, Squier, soit autant de gages du sérieux des questions qui seront débattues à Nancy. Force est de constater que Léon de Rosny et Madier de Montjau tiennent absolument à ce que cette réunion soit une pleine réussite. C'est pourquoi, d'une part, ils incitent les membres des deux sociétés à soutenir activement cette initiative et, d'autre part, ils apportent le concours déterminé des sociétés aux membres actifs du comité local d'organisation que sont Guerrier de Dumast et Adam. Ceux-ci disposent, eux-mêmes, d'un riche réseau de relations en France et dans le monde, dont ils usent pour assurer le succès de leur projet. Ils envoient des courriers à l'étranger et dans les colonies. Ils cherchent des délégués étrangers chargés de récolter les souscriptions et d'organiser l'envoi de délégations à Nancy. Ils écrivent, par exemple, au président de la République d'Haïti, à celui du Venezuela. S'adresser aux États, c'est s'assurer un gage de respectabilité et un soutien officiel. Francisco Pimentel, par exemple, illustre le concours des éminences scientifiques des pays étrangers. Il est, en effet, le plus influent des indianistes mexicains. C'est pourquoi

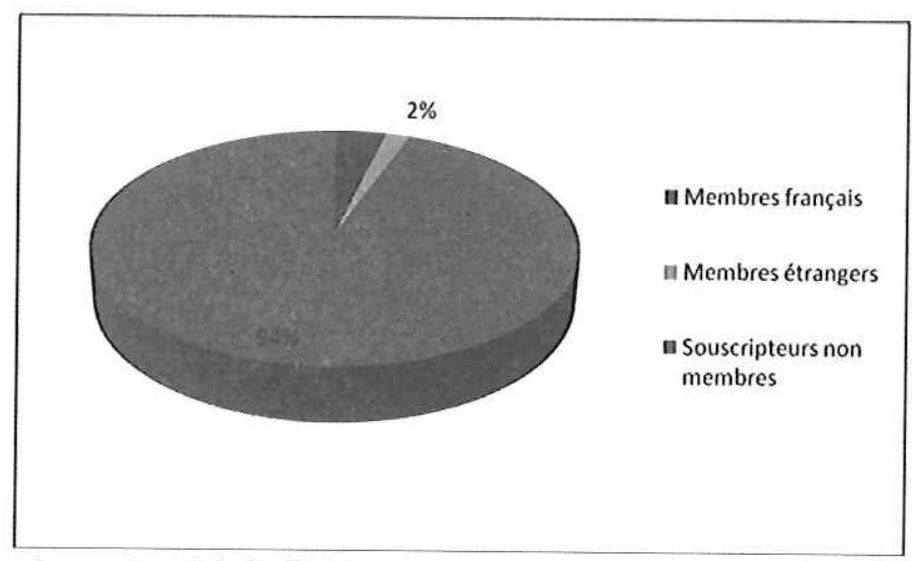

FIG. 3 - Part des membres de la Société Américaine de France dans les souscriptions - hors Lorraine. 
il accepte sa nomination comme délégué. Son nom a été proposé, comme pour d'autres délégués. Ceux-ci s'engagent donc à rechercher des souscripteurs, voire des participants, à la réunion internationale et à en assurer la promotion. Lagier, délégué de la Suisse, utilise les grands quotidiens nationaux, comme la Confédération, pour vanter les mérites du congrès de Nancy et susciter des adhésions. Le comité canadien publie, quant à lui, à ses frais, des encarts dans la presse nationale. En Équateur, une commission spéciale se réunit pour désigner un représentant qui se rendra à Nancy. Des magistrats équatoriens se chargent de traduire en espagnol les documents comme le programme du congrès. Enfin, les délégués étrangers ont souvent recours aux journaux officiels pour diffuser leurs annonces relatives à l'événement. C'est le cas en Équateur, en Guyane ou à Saint-Pierre-et-Miquelon. Ces pratiques tendent à montrer que cette manifestation, vue de l'étranger ou hors de la métropole, concerne avant tout l'élite sociale et intellectuelle. Une étude réalisée sur les souscripteurs péruviens le démontre clairement : le congrès est perçu comme un événement vecteur de modernité et de civilisation, auquel il convient de contribuer si l'on veut être intégré dans cette mouvance « éclairée »- même si l'on n'y prend aucune part active (Riviale 1989).

Les actions menées par Guerrier de Dumast et Adam les conduisent aussi à rencontrer intellectuels et hommes politiques. Le baron est en relation avec le président Mac-Mahon, des ministres comme Falloux, sans oublier les membres de l'Institut. La présence d'Adam permet d'utiliser les réseaux d'amitié des magistrats dans toute la France. Victor Hugo, ami de Burnouf, adresse au comité de Nancy sa demande d'adhésion et son soutien en janvier 1875. La liste des souscripteurs montre son succès auprès des orientalistes, américanistes ou savants d'autres disciplines comme Carnot, Castaing, le général Doutrelaine, Quatrefages, Henri Poincaré ou le jeune Vidal de la Blache.

\section{Un congrès fondateur pour les études américanistes}

La question du peuplement de l'Amérique marque profondément le congrès de Nancy : les quatre thèmes retenus pour chacune des séances se rapprochent tous de cette question. Mais celle-ci dépasse le simple cadre des recherches américanistes puisqu'elle se trouve au cœur des débats internes de la Société d'Ethnographie et, plus largement, de l'anthropologie depuis la fin du XVIII ${ }^{\mathrm{e}}$ siècle.

Dès la première journée, apparaît l'opposition de deux grands courants de pensée avec, d'un côté, les partisans d'un peuplement exogène, de l'autre, ceux d'un peuplement endogène. La lecture commentée par Léon de Rosny d'un compte rendu de recherche de Foucaux, orientaliste réputé, lance la controverse. Le travail de Foucaux porte sur «les relations qu'ont pu avoir ensemble, au commencement de notre ère, les bouddhistes d'Asie et les habitants de l'Amérique " ${ }^{11}$. Selon cet auteur, la filiation entre les religions américaines et le bouddhisme reste à prouver. Il estime que le peuplement de l'Amérique est 
endogène (ou autochtone) et, donc, que les peuples précolombiens ont connu une évolution qui leur est propre. Cela implique aussi qu'il est inutile de rechercher des liens entre les civilisations européenne, égyptienne et celles de l'Amérique. Fort de sa longue expérience de vie parmi les Indiens du Canada et, bien entendu, de ses convictions religieuses, le R. P. Émile Petitot réagit assez vivement à ces thèses. Pour lui, ces liens entre l'Amérique et l'Asie existent, comme il compte le démontrer au congrès à travers ses communications sur les Esquimaux. Le peuplement de l'Amérique est donc exogène, plus précisément par le biais de migrations issues d'Asie. Eugène Dally, président de la Société d'Anthropologie de Paris et fervent défenseur d'un matérialisme scientifique, fait immédiatement remarquer que la méthode scientifique doit impérativement être appliquée à la science américaniste. Il estime que, faute de documents attestant des relations entre l'Europe et l'Amérique avant Colomb, celles-ci doivent être considérées comme inexistantes. Il avance ensuite que les thèses anthropologiques conduisent à estimer que les Américains ne proviennent ni d'Europe, ni d'Asie, mais de leur propre continent. Petitot rétorque qu'il existe des preuves montrant l'existence des migrations asiatiques. Il s'appuie sur les récits et les légendes des peuples nord-américains, ainsi que sur l'analogie de leurs dialectes avec des langues asiatiques. La question des origines des Américains ne peut donc pas être close si tôt. Le publiciste et diplomate colombien Torres Caicedo déclare que l'originalité des monuments et des langues d'Amérique centrale et du Sud vient perturber la thèse des migrations asiatiques. Frédéric de Hellewald, délégué de l'AutricheHongrie, estime quant à lui que la situation est plus complexe : les peuples d'Amérique du Nord formeraient une " race à part "; pour lui, si des Asiatiques sont venus en Amérique, cela n'a pu se produire que fortuitement. Il rejoint Dally : les Indiens sont des Américains, qui ont évolué en s'adaptant au milieu dans lequel ils se trouvaient. Cette thèse a l'avantage d'expliquer les différentes évolutions observées parmi les peuples précolombiens, tout en acceptant quelques migrations asiatiques sporadiques et dues au hasard. Léon de Rosny juge ces théories séduisantes, mais observe que les preuves qui les étayeraient manquent toujours. Au final, le congrès décide qu'en l'état actuel des connaissances de la science, il est impossible de trancher la question. L'affaire passionne tant, que la presse locale s'empare de la question et prend même position pour l'une ou l'autre des thèses défendues. Madier de Montjau finit par intervenir depuis Paris, après le congrès, en rappelant que les deux théories restent à explorer et que le congrès s'est refusé à trancher en faveur de l'une ou de l'autre.

De la question des origines des peuples de l'Amérique découlent d'autres communications tout aussi polémiques, notamment celles traitant de « la tradition de l'Homme blanc », ou bien de l'influence du dogme chrétien dans l'interprétation des faits historiques et scientifiques. On retrouve ici les éléments d'une querelle inscrite dans le mouvement plus large qu'est celui de l'affrontement scientifique entre évolutionnistes et créationnistes. Rappelons que la révolution 
majeure provoquée par Darwin, avec ses ouvrages L'origine des espèces (1859), suivi de La Descendance del'Homme et la sélection sexuelle (1871), reste d'actualité en 1875. Si quelques communications faites pendant cette manifestation semblent être plus d'ordre strictement ethnographique (notamment les études sur les langues et les écritures: "un manuscrit iroquois ", " déchiffrements mayas », « langues cheyenne et quichua », « alphabet bihua »...) ou bien relever de l'anthropologie physique (l'anthropologie des Antilles, les crânes colombiens), les implications possibles sur la question des origines et des relations intercontinentales y transparaissent cependant en filigrane. Ces débats se poursuivront au cours des congrès suivants. C'était en définitive la question de fond depuis les premières rencontres avec les Amérindiens quatre siècles auparavant : d'où viennent-ils ? et comment ont-ils pu, pour certains d'entre eux, développer une civilisation avancée hors de l'histoire de l'Ancien Monde? Les études américanistes demeureront imprégnées de ces questions jusqu'au début du $\mathrm{xx}^{\mathrm{e}}$ siècle.

\section{Lemusée américain de Nancy}

Le congrès tenu à Nancy doit s'accompagner d'une exposition d'antiquités américaines. Une annonce officielle parait dans la presse dès la fin du mois d'octobre 1874, ce qui montre que cette idée s'est vite dessinée pour la SAF et la Société d'Ethnographie ${ }^{12}$. Cette exposition vient nourrir des desseins plus ambitieux : créer le musée américain depuis longtemps espéré (Riviale 1995, p. 221). On l'a souvent dit, le $\mathrm{xIX}^{\mathrm{e}}$ siècle est le siècle des musées : chaque municipalité, chaque société savante digne de ce nom se doit d'ouvrir son musée. Médium scientifique et pédagogique, ce type d'institution est également une marque de reconnaissance et un signe de distinction (Georgel 1994). Les congrès ne font pas exception à cette règle : les précédents congrès des orientalistes avaient organisé des expositions d'artefacts (et, sans doute aussi, de sources écrites). Dans le cas du congrès des américanistes, intervient une autre donnée : dès sa reformation en 1873, la SAF avait publié un manifeste dans lequel on proclamait le projet de créer - hors de Paris, lieu de la science académique désormais honni - un ensemble de musées dédiés aux sociétés amérindiennes (Annuaire de la Société Américaine de France, II, 1873, p. 159). Nancy offrait un cadre parfait pour réaliser ce projet. En juin 1875, Adam confirme à Léon de Rosny que la ville de Nancy est prête à accepter les ouvrages relatifs à l'archéologie américaine, ainsi que les collections d'archéologie américaine de la Société d'Ethnographie, à la condition que celles-ci soient destinées à former les premiers éléments d'une "Bibliothèque américaine et d'un Musée d'archéologie américaine » (Actes de la Société d'Ethnographie, 1874-1875, séance du 5 juillet 1875). La ville de Nancy ne veut donc pas que la bibliothèque et le Musée américain soient confondus avec d'autres établissements de la ville ; ils doivent avoir une identité propre. Nous supposons donc que l'idée de transformer l'exposition en un Musée américain a probablement pris forme en mai-juin 1875. 
Enfin, Jules Renauld, américaniste nancéien dans les années 1875-1885, explique que le musée a été créé pendant la tenue du congrès de Nancy (Renauld 1880, p. 203). La SAF a dû inciter à la création d'un Musée américain à mesure que le succès du congrès et de l'exposition d'antiquités américaines s'est confirmé. Il s'agit, pour cette société, d'élever les études américanistes au rang d'une science digne d'intérêt, au moins au même titre que les études orientalistes. Pour la ville de Nancy, Adam et Guerrier de Dumast y voient aussi une belle occasion de se positionner en capitale culturelle et scientifique de province, capable de concurrencer Paris. Le comité local d'organisation fut donc chargé de réunir les collections présentées dans l'exposition. Ainsi, Adam parvient-il à obtenir le prêt de la nouvelle collection d'antiquités caraïbes du Guadeloupéen Noirtin. La Société d'Ethnographie apporte sa contribution à la formation de la collection de l'exposition par le prêt de momies péruviennes; elle offre en outre une idole en jade. Léon de Rosny met aussi à disposition un codex maya et des masques de pierre du Mexique. Le 5 juillet 1875 , le bureau de la société propose de faire don à la ville de Nancy, outre des ouvrages d'archéologie précolombienne, « d'antiquités américaines lui [à la Société d'Ethnographie] appartenant ». Puis elle « invite ses membres à enrichir par des dons les collections offertes par la Société ", aux conditions mentionnées précédemment (Actes de la Société d'Ethnographie, 1874-1875, séance du 5 juillet 1875). En supportant ce musée américain à Nancy, la Société d'Ethnographie voit sans doute en cette nouvelle institution la possibilité de prendre le relais du musée américain du Louvre, fermé au public depuis des années (Guimaraes 1994; Riviale 1995). C'est ce que déclare triomphalement André de Clavery (1875, p. 380) :

Elle serait la meilleure collection de ce genre au monde, la mieux gardée et la mieux étudiée, la plus connue. Elle sera promptement plus utile que celles que possèdent les grands établissements de Paris, qui semblent avoir pour mot d'ordre de cacher leurs trésors, de les dédaigner, de les dilapider souvent, et d'empêcher les progrès de la science américaine.

Le contenu de l'exposition d'antiquités américaines est décrit dans les actes du congrès (Actes du Congrès international des Américanistes, 1875, pp. 21-26). On a déjà évoqué quelques dons faits par la Société d'ethnographie ${ }^{13}$, d'autres collections viennent s'y ajouter, en particulier la fameuse collection de l'orientaliste et homme d'affaires, Henri Cernuschi. La seule source sur le fonds connue jusqu'à présent était le catalogue réalisé par Jules Renauld. Il l'a présenté au congrès de Bruxelles de 1879, il y décrit d'une façon assez détaillée les 58 poteries et les deux " idoles » considérées comme appartenant à la collection du Musée américain de Nancy. Ce travail s'accompagne de quelques planches réalisées par Renauld. Certaines publications mentionnent une collection de 80 poteries, mais il s'agit en fait d'une erreur d'interprétation du préambule du catalogue de Renauld ${ }^{14}$. 
La collection offerte par Cernuschi se compose d'un grand nombre de poteries, provenant du musée Cavaleri de Milan, qu'il acquit en totalité pour la somme de 300000 lires en avril 1873. L'avocat Michele Cavaleri avait ouvert en 1871 un musée privé dans la maison Fortis à Milan. Dans cette riche collection, se trouvait un lot de poteries péruviennes ${ }^{15}$. En tant que membre de la Société d'Ethnographie, ami de Léon de Rosny et participant au premier congrès des orientalistes à Paris en 1873, Cernuschi souhaita probablement soutenir la constitution d'un musée américain et encourager son succès. Hormis ces poteries, quelques pièces sont intéressantes : un lot de moulages et une statuette en terre cuite. Le descriptif de l'exposition d'antiquités américaines mentionne le moulage d'un masque taillé dans un pétrosilex provenant de la région de Oaxaca. L'original appartenait à Eugène Boban ; une note précise qu'il est identique à l'un des masques présentés par Lucien de Rosny dans les Archives de la SAF en 1875. Dans la même vitrine, se trouvaient d'autres pièces fournies par Boban : « une collection de moulages représentant des têtes d'idoles en terre cuite, trouvées dans les environs de la Vera-Cruz», ainsi que le "moulage d'un ocelotl, ou tigre mexicain », dont l'original proviendrait de la région de Puebla. La presse lorraine signale, à la mi-juin 1875, les dons effectués par Boban à la ville de Nancy : il cédait une partie des collections mises à disposition de l'exposition (en l'occurrence ces moulages). Enfin, outre l'idole "péruvienne " en jade aux yeux d'émeraude offerte par la Société d'Ethnographie, on observe la présence dans la collection initiale d'une seconde figurine en terre cuite, d'origine péruvienne, dont le donateur est encore inconnu ${ }^{16}$. Une lettre de Lucien Adam de 1877, publiée dans les actes du congrès de Luxembourg, évoque aussi la SAF parmi les donateurs. Enfin, le Progrès de L'Est du 22 juillet 1875 nous apprend que Paul Broca venait « d'envoyer au Musée américain de Nancy des momies et les produits de l'industrie ancienne ». Nous ne disposons pas d'informations plus précises à ce jour sur ces deux donations.

Comment s'est poursuivie la vie du Musée américain de Nancy après la clôture du congrès? On dispose de très peu de sources concernant ce sujet. Les archives du Musée lorrain nous apprennent juste que Jules Renauld emporta les collections chez lui pour les étudier en vue de réaliser un catalogue qu'il comptait présenter au congrès des américanistes à Bruxelles en 1879. À partir de là, nous n'avons trace que du lot étudié par Renauld. L'édition de 1895 du catalogue des collections du Musée lorrain, établi par le conservateur Lucien Wiener (1895), montre que certains de ces objets sont exposés au public. À cette époque, le Musée lorrain s'agrandit afin de pouvoir présenter davantage de collections. Un plan manuscrit de cette époque, retrouvé dans les archives du musée, illustre un projet d'organisation des nouvelles salles. On lit dans la légende : « antiquités péruviennes ». Cela correspond, à en croire le plan, à une seule vitrine. L'histoire des autres donations demeure inconnue. Il semble que la disparition de la Société d'Études Américaines de Nancy, dont le but était 
notamment de promouvoir le musée américain de la ville, ait entraîné un désintérêt pour ces collections devenues encombrantes. Au début des années 1930 s'ouvre sous l'impulsion de Lucien Cuénot, un ami de Goury, l'Institut de zoologie, qui deviendra quelques décennies plus tard le Muséum aquarium de Nancy. Le lot péruvien y est transféré, signe de l'indifférence dans laquelle cette collection est alors tombée - à l'image du reste des rêves américains de Nancy.

\section{Après Le Congrès de NanCY}

\section{La pérennisation des congrès}

Le succès du congrès des américanistes de Nancy débouche sur la tenue d'une deuxième session à Luxembourg en 1877. La décision est prise pendant le premier congrès. Le rythme biannuel devient la règle. La possibilité de débattre des questions essentielles de la recherche américaniste et de mettre fin aux faux débats concernant les relations antiques entre l'Ancien et le Nouveau Monde, est finalement acquise à l'issue de la troisième session en 1879, à Bruxelles. Lors du cinquième congrès, le Mexique émet le souhait de voir siéger en Amérique la réunion suivante. Pour des raisons pratiques, mais surtout parce que l'Europe hésite à déplacer un congrès qu'elle ne juge pas encore assez enraciné dans le paysage scientifique, la décision est reportée à la session suivante (à Turin). C'est en 1895, pour la onzième session, que le congrès international des américanistes se rend pour la première fois en Amérique, dans la ville de Mexico.

\section{Un champ de recherche qui s'affirme}

Les cinq premières sessions sont marquées par la question de l'âge des civilisations précolombiennes. On observe de la part des américanistes une ouverture sur les sciences de leur temps, en particulier vers la géologie et la paléontologie. Ces dernières font reculer à plusieurs dizaines de milliers d'années l'âge de l'humanité européenne. On s'interroge alors pour déterminer si cette nouvelle chronologie correspond aux peuples américains. Les dogmes théologiques se retrouvent encore en opposition avec les progrès de la recherche scientifique : la chronologie biblique contre celle des strates géologiques. Dans le même temps, le vocabulaire scientifique se précise. À Copenhague (1883) est fixé l'usage systématique de " précolombien » ou d'« antécolombien » lorsqu'il s'agit de parler des anciens peuples et civilisations d'Amérique. Le sujet du peuplement de l'Amérique, âprement débattu à Nancy, continue d'alimenter les interrogations lors des réunions suivantes, avec toutefois des approches qui se veulent plus scientifiques et des choix de sources plus fiables. Les études linguistiques et philologiques continuaient à être discutées, mais dans le même temps les travaux archéologiques et ethnographiques de terrain se font plus présents ; de même, les 
problématiques de recherche se précisent et l'on énonce la volonté d'une rigueur scientifique plus marquée, tant dans les méthodes de collecte des informations que dans leur processus d'analyse et dans la construction du discours. Ainsi, l'américanisme émerge peu à peu comme champ de recherche spécifique, avec ses références et ses propres méthodes.

\section{L'échec des projets américanistes de Nancy}

Lucien Adam décide de poursuivre l'œuvre du congrès tenu à Nancy et fonde la Société d'Études Américaines de Nancy (SEAN) le 16 juin 1877. Ses finalités sont définies dans l'article 2 de ses statuts : « encourager par tous les moyens les études américaines et spécialement garder et conserver les objets qui ont déjà été donnés lors du premier congrès des américanistes tenu dans cette ville en 1875, ainsi que ceux qui pourraient être donnés par la suite ${ }^{17}$. Le but premier de cette société est donc le développement du musée américain. D'ailleurs, l'article 3 de ses statuts précise que la SEAN doit veiller à ce que le mobilier archéologique qu'elle possède ne soit pas confondu avec d'autres collections. Cependant, il ne faut pas penser que cette société abandonne pour autant ses projets initiaux. Ses membres continuent d'œuvrer dans ce sens, à l'image d'Adam lors des congrès de 1877 et de 1879.

Le travail de Jules Renauld sur les céramiques péruviennes, présenté à la session de Bruxelles, et l'activité débordante de Lucien Adam, présent aux cinq premiers congrès des américanistes, marquent la vie de l'association. Celle-ci se manifeste aussi et surtout par l'échec des grands projets - probablement inspirés par la $\mathrm{SAF}$ - qu'elle était censée soutenir : créer une chaire à la faculté de lettres et une bibliothèque américaine. Lucien Adam demeure le seul membre vraiment actif de la Société. Il poursuit son infatigable travail sur les langues qu'il présente à chaque congrès auquel il participe ${ }^{18}$. En juin 1879, Dumast félicite le conservateur du Musée lorrain de Nancy Henri Lepage ${ }^{19}$ pour avoir mis à disposition un local et une vitrine pour y déposer les collections de la société, mais il semble qu'à partir de 1880 les activités de l'association déclinent rapidement, pour s'éteindre officiellement le 12 juin 1895.

L'échec de ce projet est, en partie, lié à l'absence de personnalités jouant un rôle moteur au sein de la société. Guerrier de Dumast décède en janvier 1883, tandis que Lucien Adam part à Rennes exercer ses nouvelles fonctions de président de Chambre en décembre 1883. En outre, il convient de noter qu'Adam - l'un des seuls vrais américanistes nancéiens - fut lui-même « lâché » par la SAF. Léon de Rosny écrivait ainsi à Marcos Jiménez de la Espada ces mots sévères à l'encontre de Lucien Adam :

Le seul [sujet] qui me préoccupe, c'est de savoir si le congrès deviendra une réunion d'américanistes autorisés, ou s'il sera une tribune ouverte à quiconque voudra, sans études préalables sérieuses, lire une fantaisie quelconque sur le Nouveau Monde. 
L'américanisme est trop discrédité dans nos académies et dans nos corporations vraiment savantes, pour qu'il nous soit possible de nous asseoir plus longtemps à des réunions aussi creuses que bruyantes, dans lesquelles les travaux de valeur ne sont pas distingués des travaux sans base ni solidité [...]. J'ai eu un tort dans ma carrière, [...] c'est en voyant combien était peu nombreux le nombre de travailleurs dans le champ de l'américanisme, de vouloir inventer des américanistes. J'en ai malheureusement inventé plus d'un, et pour ne parler que de $\mathrm{M}$. Adam qui l'a reconnu au congrès de Nancy, je me berçais de l'espoir qu'avec son intelligence et sa remarquable activité, il deviendrait bientôt un collaborateur sérieux, dans une branche quelconque de notre domaine. Au lieu d'apprendre une langue américaine, $\mathrm{M}$. Adam a préféré s'occuper de $32 . .{ }^{20}$

À cela s'ajoute l'absence de projet scientifique à long terme. Ainsi, le projet muséographique est conçu sans la moindre perspective de recherche. De même, l'absence d'enseignement universitaire américaniste empêche, dans une ville de province, de susciter l'intérêt et d'attirer de nouvelles collections. Le manque d'engagement politique et/ou le manque de moyens financiers renforcent les difficultés. La bibliothèque voit ses ouvrages disséminés dans diverses institutions nancéiennes: Société d'Archéologie de la Lorraine, bibliothèque publique de Nancy, Académie de Stanislas. Ces deux dernières finissent par fusionner au $\mathrm{xx}^{\mathrm{e}}$ siècle. Comme d'autres projets provinciaux, celui de Nancy disparaît au moment où s'ouvre à Paris le Musée d'Ethnographie du Trocadéro.

\section{CONCLUSION}

Le congrès de Nancy peut être considéré comme l'un des actes fondateurs de l'américanisme. L'initiative de cette manifestation reposait essentiellement sur la nécessité d'ériger les études américanistes au rang de science pour exister, mais aussi sur des arguments plus politiques. La mobilisation d'énergies convergentes tant au niveau local, national qu'international a permis de réussir ce pari qui a priori paraissait démesuré. Certes, la plupart des projets liés à la session inaugurale ont échoué, à l'exception de la pérennisation de ces réunions internationales et de la richesse de leurs débats. C'était déjà un grand pas fait pour l'institutionnalisation de l'américanisme. Avec des objectifs assez différents, la Société Américaine de France, la Société d'Ethnographie et les élites nancéiennes ont conjugué leurs efforts et leurs réseaux pour initier une manifestation scientifique qui, après cette première réussite, devait bientôt leur échapper pour vivre sa propre vie et évoluer vers ce que l'on connaît maintenant. *

* Manuscrit reçu en juin 2008, accepté pour publication en juin 2009. 


\section{NOTES}

1. L'idée est clairement énoncée dans une plaquette résumant les premiers résultats du congrès des orientalistes : "Ces congrès ont valu à la science un autre avantage dont on avait tort de ne pas tenir compte. Les princes et les peuples ont appris à vénérer les savants et à les seconder du concours de leur puissance » (Duchateau 1874, p. 3).

2. Fondée par arrêté du 24 avril 1859, la Société d'Ethnographie Orientale et Américaine avait été formée par des personnalités issues d'horizons très divers et n'ayant pas forcément les mêmes centres d'intérêt. C'est sans doute ce qui explique qu'au fil du temps cette association ait institué différentes sections qui, en fonction de circonstances favorables ou non, proclamaient leur autonomie ou bien finissaient par réintégrer le giron de la société-mère. Pour donner une idée de ce foisonnement d'initiatives, mentionnons le Comité d'Archéologie américaine (1863), transformé après des années de silence en Société Américaine de France (1873) ; l'Athénée oriental (1864), subdivisé en 1873 en deux sections, l'une conservant ce nom tandis que l'autre prenait celui de Société d'Études japonaises, chinoises, tartares et indo-chinoises. D'autres sous-sections feraient par la suite leur apparition, puis leur sécession : le Divan oriental et africain (1880), la Société Sinico-japonaise et la Société Océanienne (1889). L'association initiale qui, quant à elle, avait en 1864 simplifié son nom en Société d'Ethnographie, donna naissance en 1875 à une Institution ethnographique, devenue en 1886 l'Alliance scientifique universelle. Il convient cependant de souligner que, derrière cette multiplicité de créations institutionnelles, se trouvait un nombre relativement restreint de membres, ce qui explique l'extrême complexité et imbrication de leurs initiatives et publications respectives.

3. Actes de la Société d' Etlhographie, 1874-1875, p. 40.

4. Sur les relations étroites entre orientalisme et américanisme dans ces années-là (et principalement au sein de la Société d'Ethnographie), voir Prévost 2007.

5. L'auteur était alors président de l'Athénée oriental. Notons également que le « règlement des sessions provinciales ", inclus dans cette même publication, est signé par Léon de Rosny.

6. Actes de la Société d'Ethnographie, 1874-1875, p. 43.

7. Burnouf est titulaire de la chaire de langues orientales à la Faculté de Nancy. Il est, avec Leupol, l'auteur d'ouvrages sur le sanscrit.

8. La disparition de l'Empire le 4 septembre 1870 précipite la fin de cette commission.

9. Après l'annonce de la contribution de Mac-Mahon, le baron Guerrier de Dumast écrit le 3 mai 1875 au ministère de l'Instruction publique pour solliciter une aide financière, qui se matérialisera par un don de 100 francs (Archives nationales, 1875, F/17/3092/3, dossier Nancy, Paris).

10. Si les organisateurs avaient craint un temps la réaction de la Prusse pour le choix du siège de ce congrès, celle-ci fut finalement représentée par une poignée de souscripteurs. On note également la contribution de plusieurs autres États allemands (Bavière, Bade, Saxe, Hesse), cependant il ne s'agit que d'une participation de principe. Force est de constater que l'on ne relève le nom d'aucune personnalité notable. En fait, la très grande majorité des souscriptions allemandes émane des territoires occupés (12 pour l'Alsace et 13 pour le pays messin). Il est vraisemblable que les autorités allemandes aient fortement découragé les inscrits de faire le voyage.

11. Il s'agit là du titre de la communication rédigée par Foucaux.

12. Le Progrès de l'Est, 30 octobre 1874.

13. Si la figurine en jade aux yeux d'émeraude, mentionnée dans les documents, paraît avoir aujourd'hui disparu, les recherches menées dans les réserves des musées nancéiens ont permis de retrouver un moulage de masque correspondant à celui offert par Léon de Rosny.

14. Pour réaliser son catalogue, Renauld $(1880$, p. 202) s'est rendu au Louvre où il a étudié 80 poteries péruviennes similaires à celles de Nancy.

15. Longtemps enfouies dans les réserves du Muséum aquarium de Nancy (MAN), ces céramiques péruviennes furent retrouvées en 2006 par l'équipe du service des collections de ce musée et inventoriées. Grâce à leur aide, il a été possible de réaliser une étude rapide des poteries. D'abord, elles ne sont 
pas toutes issues de la collection Cavaleri. Seules celles portant une étiquette manuscrite rédigée en espagnol semblent en provenir. La collection Cernuschi regrouperait alors 44 poteries. Les 14 restantes émanent d'un autre fonds restant à identifier. Dans l'ensemble, ces poteries funéraires sont assez bien conservées, bien que certaines aient été recollées afin de pouvoir être présentées au public. Une partie du lot semble de facture Mochica et les autres Chimu. Il est intéressant de signaler que les vases de la collection Cernuschi forment un ensemble assez homogène ; des étiquettes, déjà considérées comme " très anciennes » par Renauld (1880), rédigées en espagnol, donnent des indications de provenance très précises (en majorité des huacas des environs de Casma, Pacasmayo, Chota et Trujillo). Cette série de vases fut par la suite, vers 1930, mise en dépôt à l'Institut de zoologie. L'histoire de ces collections reste marquée par la question épineuse de leur propriété : à ce jour, personne ne sait vraiment qui en jouit, d'où le statut de dépôt provisoire qui leur a été réservé lors de l'enregistrement sur l'inventaire du MAN en 2006.

16. Elle est aujourd'hui conservée dans les réserves du MAN. Il convient également de signaler que, dans ces mêmes réserves, se trouve une statuette péruvienne d'une dizaine de centimètres de haut, offerte par Georges Goury, ancien conservateur du Musée lorrain, responsable des estampes, livres et sceaux au début du $\mathrm{xx}^{\mathrm{e}}$ siècle.

17. Archives départementales de Meurthe-et-Moselle, 4M114, liasse Sociétés d'Études.

18. Pour une évocation plus étendue de l'œuvre linguistique de Lucien Adam, voir Desmet (1996, pp. 435-463).

19. Archives départementales de Meurthe-et-Moselle, MS484SAL/1, Correspondance Lepage, liasse correspondance Guerrier de Dumast.

20. Lettre de Léon de Rosny à Marcos Jiménez de la Espada ( $1^{\text {er }}$ novembre 1879), Archives du Consejo Superior de Investigaciones Científicas, Madrid, Papiers Jiménez de la Espada, signature 8518. Ce courrier vient peu après la clôture du congrès des américanistes de Bruxelles et se situe dans le contexte de la préparation du congrès suivant à Madrid. Rosny sent que le congrès se trouve à un tournant de son histoire et cherche des "alliés " pour y maintenir une approche qu'il considère scientifique. Ancien membre de la commission espagnole du Pacifique (1862-1866), le naturaliste Marcos Jiménez de la Espada était devenu l'une des figures marquantes de l'américanisme espagnol (notamment avec son travail de redécouverte et d'édition des chroniques de la Conquête et de la Colonie). Sur cette personnalité et ses sources, voir López-Ocón et Pérez-Montes (2000). Nous remercions Leoncio López-Ocón, du CSIC, de nous avoir révélé ce fonds important pour l'histoire de l'américanisme.

\section{RÉFÉRENCES CITÉES}

\section{Actes de la Société d'Ethnographie} 1874-1875 tome VIII.

\section{Actes du congrès international des américanistes} 1875 Crépin-Leblond, Nancy.

Adam Lucien

1874 "Le congrès international des orientalistes », Mémoires de l'Académie Stanislas [1873], $4^{\mathrm{e}}$ série, tome VI, pp. XIX-XL.

\section{Clavery André de}

1875 "Le congrès des américanistes », Archives de la Société Américaine de France, n. s., I, p. 380. 
Comas Juan

1974 Cien años de Congressos Internacionales de Americanistas, UNAM/Instituto de Investigaciones Históricas, Mexico.

Desmet Piet

1996 La linguistique naturaliste en France (1867-1922). Nature, origine et évolution du langage, Peeters, Louvain/Paris.

Duchateau Julien

1874 Une création scientifique française. Le premier congrès international des orientalistes, Dentu, libraire et $\mathrm{Mme} \mathrm{V}^{\mathrm{e}}$ Bouchard-Huzard, Paris.

Georgel Chantal (éd.)

1994 La jeunesse des musées: les musées de France au $x t x^{e}$ siècle. Catalogue de l'exposition au Musée d'Orsay, du 7 février au 8 mai 1994, Réunion des musées nationaux, Paris.

\section{Guimaraes Susana}

1994 Le Musée des antiquités américaines du Louve (1850-1887). Une vision du collectionnisme américain au $x x^{e}$ siècle, mémoire de maîtrise, Université Paris 1, Panthéon-Sorbone, Paris.

Logie Étienne

2008 "Nancy et le premier congrès international des américanistes », Le Pays Lorrain, mars, Nancy, pp. 35-40.

LóPez-Ocón Leoncio et Carmen María PéRez-Montes

2000 Marcos Jiménez de la Espada (1831-1898). Tras la senda de un explorador, Consejo Superior de Investigaciones Científicas/Instituto de Historia, Madrid.

Prévost Nadia

2007 Brasseur de Bourbourg et l'émergence de l'américanisme scientifique en France au $x x^{2}$ siècle, thèse de doctorat, Université de Toulouse II, Toulouse.

Riviale Pascal

1989 "Archéologie et sociabilité. La délégation française du Pérou au premier congrès international des américanistes, Nancy, 1875 », Bulletin de l'Institut français d'études andines, XVIII, pp. 55-64.

1995 "L'américanisme français à la veille de la fondation de la Société des américanistes ", Journal de la Société des Américanistes, 81, pp. 207-229.

1996 Un siècle d'archéologie française au Pérou (1821-1914), L'Harmattan, coll. « Histoire des Sciences Humaines », Paris.

Renauld Jules

1880 "La céramique péruvienne de la Société d’Études Américaines, fondée à Nancy », Mémoires de l'Académie de Stanislas, Berger-Levraut, Nancy.

TEXTOR DE RAVISI

1875 "Invitation ", in Comité national français du congrès des orientalistes (Athénée oriental). Congrès provincial des orientalistes à Saint-Étienne du 19 
au 25 septembre 1875. Règlement de la session de Saint-Étienne (Loire), Saint-Étienne, chez le directeur-président [brochure publiée à l'occasion de ce congrès].

WIENER Lucien

1895 Musée historique lorrain au palais ducal de Nancy. Catalogue des objets d'art et d'antiquité, Palais ducal/René Wiener, Nancy. 